\title{
Absceso subpectoral como diagnóstico diferencial de hombro doloroso - reporte de un caso
}

\section{Subpectoral Abscess as a Differential Diagnosis of Painful Shoulder - A Case Report}

\author{
José Palma Concha ${ }^{1}$ Andrés Gómez Meier ${ }^{1}$ Enrique Herrera Otto ${ }^{2}$ Juan Besser Mahuzier ${ }^{3}$
}

George Ayres Henriquez ${ }^{3}$

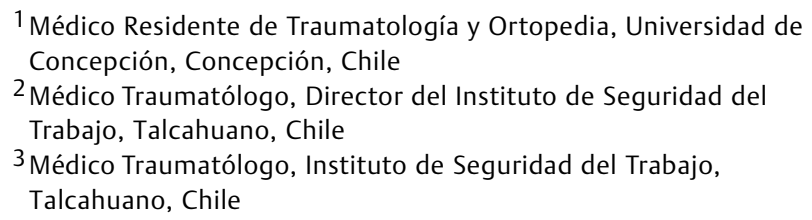

Address for correspondence Dr. José Palma Concha, Médico Residente, Instituto de Seguridad del Trabajo Talcahuano, Universidad de Concepción, San Martín 1580, Región del Bío - Bío, Concepción, Chile (e-mail: jose.palma.concha@gmail.com).

Rev Chil Ortop Traumatol 2018;59:35-39.

\section{Resumen \\ Palabras clave \\ - absceso \\ - hombro doloroso \\ - infecciones estafilocócicas \\ - manguito rotador}

\section{Abstract \\ Keywords \\ - abscess \\ - painful shoulder \\ - rotator cuff \\ - staphylococcus infection}

Los síndromes dolorosos del hombro son relativamente comunes en la práctica clínica. Habitualmente son causados por un número limitado de patologías. Dentro de los diagnósticos diferenciales, el pinzamiento subacromial, las lesiones aisladas del manguito rotador, capsulitis adhesiva, tendinitis cálcica, patología degenerativa de las articulaciones glenohumeral y acromioclavicular, y la inestabilidad crónica del hombro, son causas comunes. Causas infrecuentes son la rotura del tendón del bíceps, neuralgias, patología infecciosa articular y tumores del hombro. Un absceso subpectoral sin sintomatología infecciosa clara es una causa extremadamente rara de hombro doloroso en el adulto. Presentamos el caso de un paciente de 60 años, que inicia con un cuadro de hombro doloroso cuya causa se identifica como un absceso subpectoral por staphylococcus aureus que se maneja con drenaje quirúrgico y tratamiento antibiótico endovenoso con buenos resultados.

Painful shoulder syndromes are commonly caused by a limited assortment of pathologies. Differential diagnosis include rotator cuff impingement syndrome, rotator cuff tears, adhesive capsulitis, calcific tendonitis, degenerative disease of the joint including acromio-clavicular and gleno-humeral joints and chronic instability. Less common causes are labral tears, biceps tendon rupture, soft tissue infection, neurologic disease, joint infection and shoulder tumors. A subpectoral abscess without infectious clinical features is a very rare cause of shoulder pain in adults. We present the case of a 52 years old male who develops a painful shoulder syndrome caused by a staphylococcus aureus subpectoral abscess, treated by surgical drainage and intravenous antibiotic therapy with good results.
DOI https://doi.org/

10.1055/s-0037-1615255. ISSN 0716-4548.
Copyright $(2018$ by Thieme Revinter

Publicações Ltda, Rio de Janeiro, Brazil
License terms

(c) (i) $\ominus$ (\$) 


\section{Introducción}

Los síndromes dolorosos del hombro son relativamente comunes en la práctica clínica, con una incidencia que puede alcanzar un $66,7 \%$ en el transcurso de la vida de un individuo, siendo ligeramente mayor en mujeres. ${ }^{1}$

Habitualmente son causados por un número limitado de patologías, donde la historia clínica y el examen físico son esenciales para su adecuado enfrentamiento diagnóstico y para orientar la solicitud de exámenes imagenológicos o de laboratorio. ${ }^{2}$ Dentro de los diagnósticos diferenciales comunes que el clínico debe plantearse a la hora de evaluar a un paciente con un cuadro de hombro doloroso encontramos el pinzamiento subacromial, lesiones aisladas del manguito rotador, capsulitis adhesiva, tendinitis cálcica, patología degenerativa que puede afectar a las articulaciones glenohumeral y acromioclavicular, y por último la inestabilidad crónica del hombro. Otras causas menos comunes son roturas del tendón del bíceps, patología infecciosa articular y tumores primarios o secundarios del hombro. $^{2}$ La artritis séptica del hombro es infrecuente, presentándose en alrededor de un $5 \%$ de todos los pacientes con diagnóstico de artritis séptica. Involucra frecuentemente la articulación glenohumeral, y en casos excepcionales, la articulación acromioclavicular en forma aislada. ${ }^{3}$ La presentación clínica de las infecciones en torno a la articulación del hombro corresponde por lo general a la sintomatología clásica de fiebre, aumento de volumen, rigidez y dolor, sin embargo, hasta en un $42 \%$ de los pacientes la fiebre puede ser baja o estar ausente, ${ }^{4}$ más aún en pacientes con inmunosupresión la semiología clásica puede no formar parte de la presentación clínica del cuadro. ${ }^{5}$ La identificación de un absceso subpectoral aislado, en el contexto de un paciente sin sintomatología infecciosa clásica, es una causa extremadamente rara de síndrome de hombro doloroso, habiendo muy pocos casos reportados en la literatura. ${ }^{6-8}$ Presentamos el caso de un paciente de 60 años quien posterior a un esfuerzo físico mínimo inicia cuadro de hombro doloroso, cuya causa resulta ser un absceso subpectoral de presentación subaguda.

\section{Caso Clínico}

Paciente de sexo masculino, 60 años, con antecedentes de hipertensión arterial y diabetes mellitus tipo II, con buen control y tratamiento.

Consulta inicialmente con médico general por cuadro de tres días de evolución de dolor mal caracterizado en hombro derecho, con irradiación a brazo ipsilateral de inicio súbito luego de levantar objeto de $30 \mathrm{Kg}$. En el momento de la consulta se realizaron pruebas específicas para evaluación del manguito rotador, que incluyeron test Jobe, Patte, Gerber y Speed test, las que resultaron negativas. Se indicó realización de electrocardiograma, informado por cardiólogo como normal. Se diagnosticó síndrome de hombro doloroso derecho, indicándose antiinflamatorios no esteroidales y fisioterapia. Ante la persistencia de sintomatología, consultó en nuestro centro traumatológico. En dicha ocasión destacó normalidad en la inspección de piel circundante a la articulación del hombro y sin lesiones en la axila. A la palpación, dolor en región deltopectoral asociado a limitación en rango de movimiento activo, con flexión anterior de $160^{\circ}$, extensión $50^{\circ}$, abducción limitada a $120^{\circ}$, rotación externa dolorosa y limitada a $30^{\circ}$, rotación interna dolorosa pero completa. Examen neurovascular de la articulación del hombro normal.

Se indicó la realización de ecotomografía de la región deltopectoral derecha en la que destacó una tendinosis cálcica del subescapular y supraespinoso además de un foco de edema en relación al músculo pectoral mayor ( - Fig. 1). Se indicó continuar con fisioterapia y analgésicos.

Tres días después y ante la nula respuesta a la terapia indicada, el paciente consulta nuevamente con el traumatólogo. En el examen físico destacó paciente afebril y en buen estado general, signos vitales dentro de rangos normales. En la inspección, ligero aumento de volumen en región pectoral derecha, ausencia de lesiones en la piel circundante y axila. A la palpación región indurada y tumefacta sobre el pectoral mayor, sin calor local ni eritema, sin adenopatías cervicales ni axilares. En examen motor, persistencia de dolor en abducción y rotación externa, examen neurovascular del hombro y brazo dentro de rangos normales, en consecuencia, se solicitó tomografía axial computada de tórax en la que destacaron fenómenos inflamatorios importantes con edema muscular y de planos grasos de la región pectoral derecha de caracteres inespecíficos, que, según apreciación del radiólogo, pudieran corresponder a un fenómeno de rabdomiolisis.

El traumatólogo evaluó exámenes imagenológicos y solicitó exámenes de laboratorio entre los que destacaron: Glicemia en ayunas: $115 \mathrm{mg} / \mathrm{dL}$; Creatinina sérica: $0.88 \mathrm{mg} / \mathrm{dL} ; \mathrm{CK}: 69 \mathrm{UI} / \mathrm{L}$; LDH: 17; PCR: $51 \mathrm{mg} / \mathrm{L}$; Hemograma: Leucocitos 7.500/mL (7.500 globulos blancos por $\mathrm{mL}$ ) con distribución normal; Procalcitonina: $0.08 \mathrm{ng} / \mathrm{mL}$; VHS: $66 \mathrm{~mm} / \mathrm{hr}$.

VIH, VDRL y factor reumatoideo negativos; Ácido úrico $5.2 \mathrm{mg} / \mathrm{dL}$; Hemoglobina glicosilada 6,1\%. Se solicitó una nueva ecografía de pectoral derecho para precisar hallazgos de tomografía computada que concluyó: Colección intramuscular del pectoral mayor $10.6 \times 6.8 \times 4.6 \mathrm{~cm}$ con aspecto de hematoma (-Fig. 2). Ante nula respuesta a tratamientos indicados, luego de 11 días de evolución, se decide en acuerdo

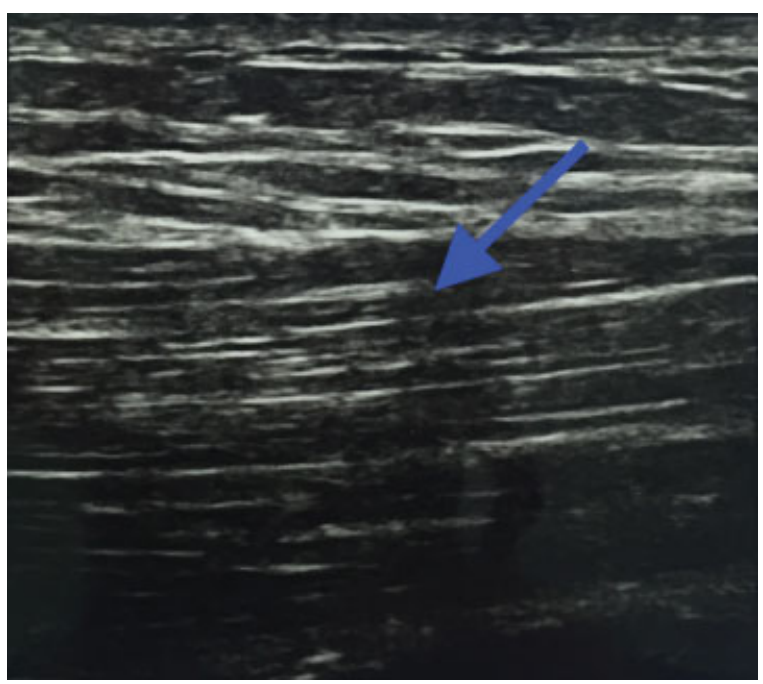

Fig. 1 Ecotomografía de región pectoral, flecha indica zona de inflamación difusa en fibras del pectoral mayor derecho. 


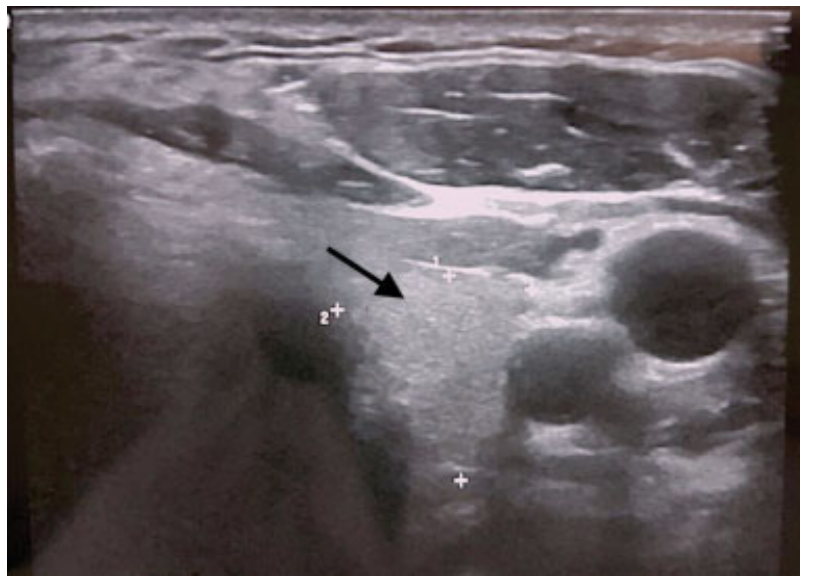

Fig. 2 Ecotomografía de región pectoral realizada posterior a lo descrito en - Fig. 1. Flecha muestra colección en fibras profundas del pectoral mayor derecho.

con el paciente, realizar tratamiento quirúrgico para evaluar colección observada en ecotomografía. Paciente ingresa a unidad de hospitalización quirúrgica de nuestra institución, se solicitaron exámenes preoperatorios entre los que destacaron: Creatinina 0.7 mg/dL; PCR: 176 mg/L; INR: 1; PT: 100\%.

Paciente persistió en todo momento afebril y con signos vitales dentro de parámetros normales. Se realizó drenaje quirúrgico, realizando incisión transversa sobre sitio de la lesión y disección por planos sin encontrarse alteraciones en planos subdérmico ni muscular (-Figs. 3 y 4), con salida de abundante material purulento desde plano submuscular del pectoral mayor. Se tomaron tres cultivos corrientes para microorganismos aerobios en intraoperatorio, se instaló

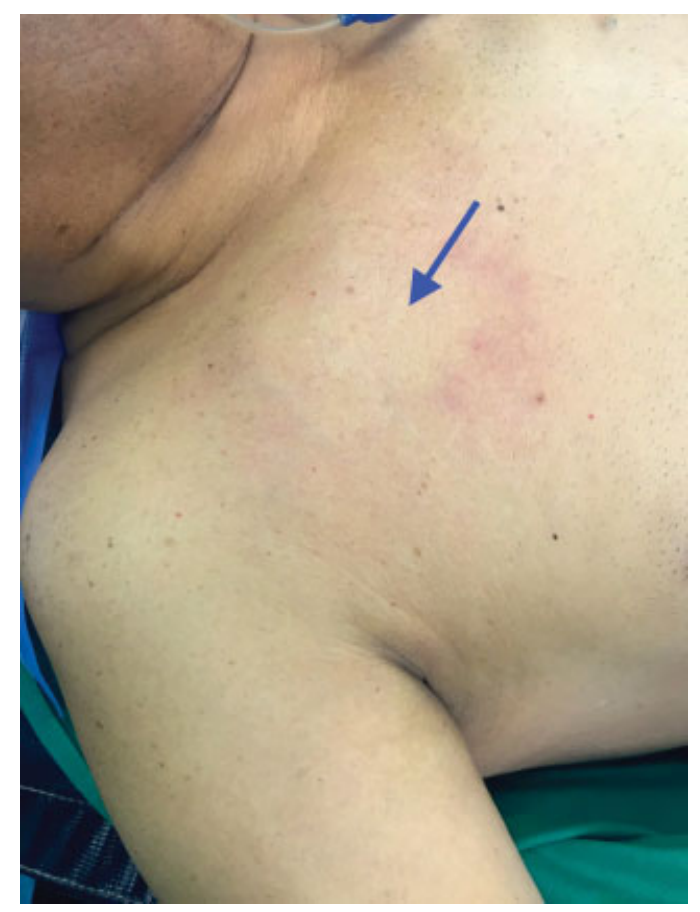

Fig. 3 Aspecto clínico de sitio de absceso en región pectoral derecha previo a drenaje, flecha indica zona de mayor induración y aumento de volumen.

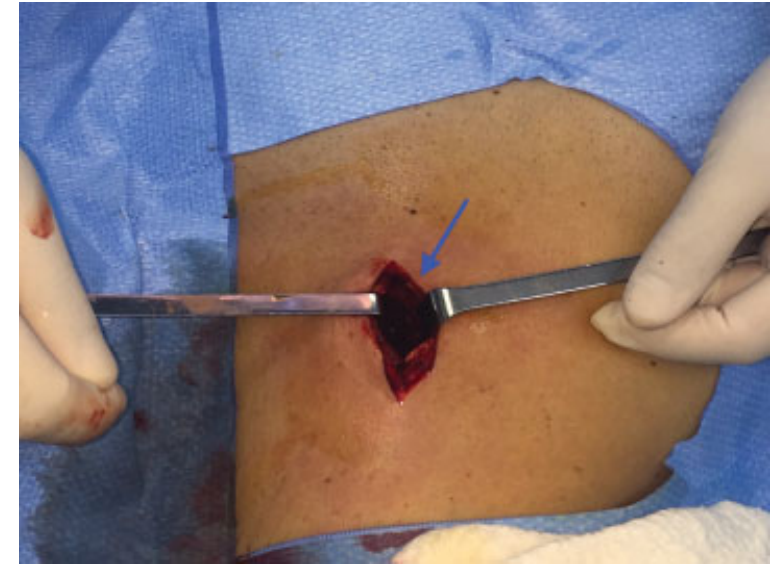

Fig. 4 Se evidencia salida de material purulento al drenar en forma quirúrgica el absceso, dejando un espacio cavitado, indicado por la flecha.

drenaje tipo penrose y se inició tratamiento antibiótico empírico para cobertura de staphylococcus aureus con cloxacilina 2 gr. Cada 6 horas por vía endovenosa. El drenaje presentó salida 20 cc de líquido serohemático luego de 24 horas de evolución, retirándose en ese momento. Al tercer día del tratamiento, se informó cultivo positivo para staphylococcus aureus sensible a cloxacilina. Se mantiene terapia con cloxacilina endovenosa durante 5 días, con disminución de parámetros inflamatorios y con excelente respuesta clínica, por lo se realiza traslape a cloxacilina oral 1 gr. cada 6 horas para completar un total de 14 días de tratamiento antibiótico. Evoluciona sin dolor, con rangos de movilidad completa en hombro derecho.

Se realiza curación en sitio de incisión para drenaje y es finalmente dado de alta del servicio de hospitalizados al séptimo día post quirúrgico. (-Fig. 5). Una semana después, el paciente no presentó recidiva de sintomatología en sitio quirúrgico y retornó a sus actividades normales.

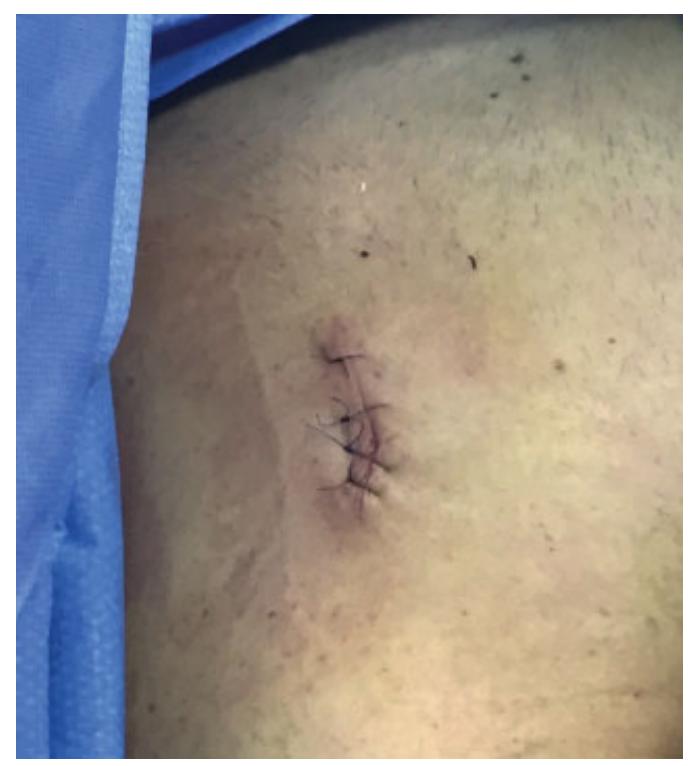

Fig. 5 Aspecto clínico de la zona intervenida al momento del alta hospitalaria del paciente. 


\section{Discusión}

La presentación clínica característica de un síndrome de hombro doloroso incluye dolor, rigidez y disminución en rangos de movimiento. ${ }^{2}$ El examen físico es indispensable y debe ser lo más completo posible. Los exámenes imagenológicos deben ser solicitados en concordancia con la clínica. En el caso de nuestro paciente, el hallazgo en la ecotomografía de tendinitis cálcica en músculos del manguito rotador, podría haber explicado su sintomatología dolorosa, tal como lo demuestra un estudio publicado en 2010 por Le Goff y col. ${ }^{9}$ En él se establece una concordancia entre la percepción de dolor y hallazgos ecográficos. Sin embargo, nuestro paciente presenta durante su evolución, algunos elementos clínicos, como la aparición de dolor e induración en la región pectoral ipsilateral que obligan a plantear diagnósticos alternativos a los ya mencionados.

Dentro de esos, encontramos los cuadros de etiología infecciosa. Si bien son poco frecuentes en la población en general, deben tenerse presentes en algunos grupos específicos como los pacientes inmunosuprimidos, los pacientes portadores de patología reumatológica o los usuarios de cortico esteroides, quienes presentan un peor pronóstico asociado a un diagnóstico tardío y dificultades en su enfrentamiento terapéutico y tratamiento. ${ }^{5} \mathrm{El}$ mayor factor de riesgo para el desarrollo de un cuadro infeccioso intrarticular corresponde al daño preexistente en la articulación. Dentro de este grupo, aparte de los pacientes ya señalados, encontramos a aquellos portadores de patologías como la gota, pseudogota, osteoartritis, lupus, cirugía reciente, trauma, diabetes mellitus y los usuarios de drogas de abuso por vía parenteral. ${ }^{3}$ El paciente presentado contaba con antecedentes de diabetes mellitus tipo II, sin embargo, sus controles eran estrictos y no contaba con otros antecedentes en su historia clínica, por lo que la realización de un screening básico de patologías que ocasionan daño articular o inmunosupresión, permitió orientar la conducta terapéutica, considerando además los hallazgos de imagenológica obtenidos mediante tomografía computada. Finalmente, los antecedentes mencionados y la visualización de material purulento durante la exploración quirúrgica de la lesión, llevaron a nuestro equipo a plantear el diagnóstico de un absceso subpectoral como la etiología probable del cuadro doloroso por el que consultó inicialmente el paciente.

El principal agente causal de infecciones de partes blandas y de patología infecciosa intrarticular en pacientes inmunocompetentes es el staphylococcus aureus sensible a meticilina. ${ }^{3}$ Su presentación clínica en patología infecciosa de tejidos blandos y articulaciones sin la sintomatología clásica descrita en la literatura, es inusual. ${ }^{4,10}$ El hallazgo de un absceso subpectoral como etiología de un síndrome de hombro doloroso es de la misma manera, extremadamente infrecuente. $^{6-8}$

Milch describe en 1928 la forma de presentación y enfrentamiento de los abscesos subpectorales, teniendo como mecanismo etiopatogénico habitual la extensión desde lesiones cutáneas que pueden ser inaparentes al examen físico. ${ }^{11}$

El enfrentamiento quirúrgico, la toma de cultivos, el inicio de tratamiento antibiótico empírico con cobertura para staphylococcus aureus y su posterior ajuste según susceptibilidad en el antibiograma, constituyen la base del tratamiento que debe plantearse para esta patología, ${ }^{12}$ tal como se realizó en el caso de nuestro paciente. Otros agentes etiológicos en este tipo de infecciones son menos frecuentes, entre ellos streptococos, como el streptococcus agalactiae y bacilos gram negativos como la Escherichia coli o pseudomonas aeruginosa, esos últimos, aislados más frecuentemente en pacientes inmunosuprimidos o usuarios de drogas de abuso por vía parenteral. El rasgo en común para el correcto abordaje de las infecciones causadas por esos agentes, es la obtención de una adecuada historia clínica y la realización de un completo examen físico, manteniendo un alto nivel de sospecha, para orientar la realización de exámenes de laboratorio e imagen. ${ }^{13}$

\section{Conclusiones}

Los síndromes de hombro doloroso pueden tener como origen patologías externas a la articulación del hombro, sin embargo esta forma de presentación es poco habitual. Destacamos la importancia de realizar un examen físico adecuado y guiar los exámenes imagenológicos y de laboratorio en función de lo anterior. El enfrentamiento de un absceso subpectoral requiere drenaje quirúrgico y cobertura antibiótica para staphylococcus aureus en forma precoz para su adecuado manejo, evitando así el riesgo de complicaciones sistémicas.

Conflictos de Interés

Sin conflictos de interés.

Fuente de Financiamiento

Sin financiamiento externo.

\section{Referencias}

1 Luime JJ, Koes BW, Hendriksen IJ, et al. Prevalence and incidence of shoulder pain in the general population; a systematic review. Scand J Rheumatol 2004;33(02):73-81. Doi: 10.1080/03009740310004667

2 Frost A, Robinson C. The painful shoulder. Surgery 2006;24(11): 363-367. Doi: 10.1053/j.mpsur.2006.09.007

3 Ross JJ. Septic Arthritis of Native Joints. Infect Dis Clin North Am 2017;31(02):203-218. Doi: 10.1016/j.idc.2017.01.001

4 Weston VC, Jones AC, Bradbury N, Fawthrop F, Doherty M. Clinical features and outcome of septic arthritis in a single UK Health District 1982-1991. Ann Rheum Dis 1999;58(04):214-219. Doi: 10.1136/ard.58.4.214

5 Edwards SA, Cranfield T, Clarke HJ. Atypical presentation of septic arthritis in the immunosuppressed patient. Orthopedics 2002;25 (10):1089-1090

6 Park GY, Kwon DR, Kim JK. Subpectoral abscess with anterior chest pain caused by Staphylococcus aureus. Am J Phys Med Rehabil 2014; 93(05):456-457. Doi: 10.1097/PHM.0b013e318278a5c7

7 Angadi DS, Nagappa S, Simpson D, Morgan I. Streptococcus subpectoral abscess with shoulder pain: A rare emergency with a common symptom? Int J Shoulder Surg 2010;4(01):22-23. Doi: 10.4103/0973-6042.68419

8 Abuelreish MA, Rathore MH. Subpectoral abscess a rare group A beta-hemolytic Streptococcus infection. Pediatr Infect Dis J 2005; 24(12):1121-1122 
9 Le Goff B, Berthelot JM, Guillot P, Glémarec J, Maugars Y. Assessment of calcific tendonitis of rotator cuff by ultrasonography: comparison between symptomatic and asymptomatic shoulders. Joint Bone Spine 2010;77(03):258-263. Doi: 10.1016/j.jbspin.2010.01.012

10 Bouvet C, Gjoni S, Zenelaj B, Lipsky B, Hakko E, Uçkay I. Staphylococcus aureus soft tissue infection may increase the risk of subsequent staphylococcal soft tissue infections. Int J Infect Dis. May 2017. Artículo aceptado, no publicado. Consultado: el 18 de Mayo 2017. Disponible en: http://www.ijidonline.com/article/ S1201-9712(17)30138-8/pdf
11 Milch H. Acute subpectoral abscess. Ann Surg 1928;87(04): 517-527

12 Weber Z, Ariano R, Lagacé-Wiens P, Zelenitsky S. Multifaceted antibiotic treatment analysis of methicillin-sensitive Staphylococcus aureus bloodstream infections. Int J Antimicrob Agents 2016;48(06): 674-679. Doi: 10.1016/j.ijantimicag.2016.09.007

13 Dubost J, Couderc M, Tatar Z, et al. Three-decade trends in the distribution of organisms causing septic arthritis in native joints: single-center study of 374 cases. Joint Bone Spine 2014;81(05): 438-440. Doi: 10.1016/j.jbspin.2014.05.001 